\title{
The Prognostic Value of Lymphocyte-to-Monocyte Ratio and Nutritional Index for Ovarian Cancer Patients with Normal CA125 Level
}

\author{
Songwei Feng ${ }^{1,2}$, Shanhui $\mathrm{Luo}^{2}$, Chenchen $\mathrm{Ji}^{3}$ and $\mathrm{Yi} \mathrm{Li}^{1 *}$ \\ ${ }^{1}$ Department of Gynecology, the Affiliated Jiangsu Shengze Hospital of Nanjing Medical University \& Jiangsu Shengze Hospital, Suzhou, People's Repub- \\ lic of China \\ ${ }^{2}$ Department of Gynecology, The Second Affiliated Hospital of Soochow University, Suzhou, People's Republic of China \\ ${ }^{3}$ Orthopedic Institute, Soochow University, Suzhou, People's Republic of China
}

${ }^{*}$ Corresponding author: Yi Li, Department of Gynecology, The Affiliated Jiangsu Shengze Hospital of Nanjing Medical University \& Jiangsu Shengze Hospital, 1399 Shunxin Middle Road, Suzhou 215228 Jiangsu Province, People's Republic of China

Received: January 12, 2021; Accepted: January 19, 2021; Published: January 28, 2021

\begin{abstract}
Background: Ovarian Cancer (OC) cases with low CA-125 concentration during routine physical examination testing are troublesome and raise false negative findings ratio. The aim of this analysis was to determine whether the Lymphocyte-To-Monocyte Ratio (LMR) and the Nutritional Index (NI) of OC patients with normal CA-125 levels had a predictive role.

Methods: This retrospective study enrolled a total of 102 OC-diagnosed patients who underwent primary cytoreductive surgery and adjuvant platinumbased chemotherapy from 2010 to 2019. Using Receiver Operating Characteristic Curves (ROC) for survival analysis, optimum cut-off values for NI and LMR were calculated. The Kaplan-Meier (KM) curve and Cox regression determined the prognostic value for Overall Survival (OS) and ProgressionFree Survival (PFS).

Results: The results showed that the optimal cutoff values were 47.5 and 4.25, respectively, for NI and LMR. NI was shown to be significantly correlated with FIGO stage, Grade, the involvement of malignant ascites, and platinum response, and LMR with FIGO stage, lymph node metastasis, malignant ascites, and platinum response when the population was separated using optimized cut-off. The 5-year OS and PFS were greatly enhanced by a high NI ( $\geqq 47.5)$. A low LMR $(<4.25)$ was associated significantly with poor 5-year PFS and OS. Both NI and LMR were independent prognosticators for the 5 -year OS in multivariate analysis.
\end{abstract}

Conclusions: In CA125-normal ovarian cancer cases, elevated NI and LMR are positive prognosticators.

Keywords: Ovarian cancer, Lymphocyte-To-Monocyte Ratio (LMR), Nutritional Index (NI), Prognosis; CA125

\section{Introduction}

One of the leading causes of cancer-related mortality in women, Ovarian Cancer (OC) accounts for 295,000 new cancer cases and 185,000 deaths worldwide annually. Among malignant gynecological tumors, the OC mortality rate is the largest, which severely endangers the health of women [1]. CA-125 is the gold standard tumor marker and has thoroughly been studied in OC [2]. For OC screening and clinical evaluation help, thirty-five kilounits/L is the cut off value of serum CA125 concentration [3]. However, during the clinical examination for OC screening, not all OC patients show perfect testing outcomes. Around $20 \%$ of women with OC have serum CA125 concentrations smaller than 35 kilounits/L [4]. Elevated false negative findings are obtained during OC screening due to these low CA125 concentration cases, which do not facilitate early diagnosis of OC.

Inflammation increases the risk and development of cancer, including initiation, promotion, malignant conversion, invasion, and metastasis [5-7]. It is considered to play an important role in tumorigenesis. Recent studies have shown a negative prognostic value of higher neutrophil-to-lymphocyte ratio and lower Lymphocyte-ToMonocyte Ratio (LMR) in OC patients, suggesting that low LMR is an independent survival prognostic factor in OC patients [8]. However, the predictive role of LMR in ovarian cancer with low CA125 concentration has not been explored.

Nutritional impairment has also been shown to have a detrimental effect on clinical outcomes [9]. At the time of diagnosis, patients with OC are also subject to starvation because of inadequate nutritional consumption due to cancer-related discomfort or psychiatric issues [10]. The prognostic Nutritional Index (NI), measured as mentioned above, could be particularly useful since both inflammation and nutritional status may serve as a surrogate marker [11]. To demonstrate the connection between postoperative complications and prognosis in patients with esophageal carcinoma [12], this index was originally examined. In this study, a low NI was seen as a poor survival predictor. 
However, the predictive role of NI in ovarian cancer with low CA125 concentration has not been explored.

Based on these observations, it seems urgent to determine a way to prevent false negative results due to low CA125 concentration. The objective of this research was to determine the effects of NI and LMR on OC patients with low CA125 concentration.

\section{Materials and Methods}

\section{Patient population}

The research approved by Ethics Committee of Soochow University retrospectively enrolled a total of 102 OC patients who underwent primary debulking and adjuvant paclitaxel and carboplatin chemotherapy in university hospitals between January 2010 and January 2019. Due to potential influences on laboratory test outcomes, patients with any inflammatory disorder were omitted. No neoadjuvant chemotherapy was given to the patient. Histological diagnosis were based on WHO guidelines, and an expert pathologist examined all microscope slides. Information collection of absolute lymphocyte, monocyte counts, and albumin tests using a peripheral blood sample were performed within one week prior to treatment. Clinical variables of concern, including clinicopathological attributes, such as such as, age, FIGO stage, Grade, LN metastasis, malignant ascites, CA125 level, residual mass, Histopathology types, and platinum response were collected and evaluated, as shown in Table 1.
By dividing the baseline total peripheral lymphocyte count (cells/ $\mathrm{mm}^{3}$ ) by the absolute peripheral monocyte count $\left(\right.$ cells $/ \mathrm{mm}^{3}$ ), the LMR was computed. The NI was measured as follows: $10^{*}$ serum albumin baseline $(\mathrm{g} / \mathrm{dL})+0.005^{*}$ absolute lymphocyte baseline count (cells $/ \mathrm{mm}^{3}$ ).

\section{Statistical Analysis}

The R software x64 (version 4.0) was used to analyze the results. To evaluate variations between proportions, the Chi-square test was used, and Kaplan-Meier analysis using the log-rank test obtained the OS and PFS curves. In evaluating hazard ratios (HR) and multivariate analysis, Cox regression analysis was used. The $\mathrm{P}$-values presented are two-sided, and statistical significance was considered at $\mathrm{P}<0.05$.

\section{Results}

\section{Study Population Characteristics}

The normal concentration of CA125 was described in our study as patients with a concentration equal to or below $35 \mathrm{U} / \mathrm{ml}$. The following optimal cut-off values were identified: 4.25 for LMR (AUC $=0.748$, $\mathrm{P}<0.001$ ) and 47.5 for PNI (AUC $=0.755, \mathrm{P}<0.001$ ), as shown in Figure 1. Therefore, patients with LMR $>4.25$ were referred to HighLMR and patients with NI $>47.5$ were referred to High-NI. Among those 102 patients, the median age was 53 years. As previously stated, in our patients, early stage disease was more frequent than advanced disease, 60 patients had stage I to II, and 42 had stage III to IV disease.

Table 1: Clinical and pathologic characteristics according to NI or LMR in 102 patients.

\begin{tabular}{|c|c|c|c|c|c|c|c|c|}
\hline \multirow{2}{*}{ Variable } & & \multirow[t]{2}{*}{ All Case } & \multicolumn{2}{|c|}{ NI } & \multirow{2}{*}{ p value } & \multicolumn{2}{|c|}{ LMR } & \multirow{2}{*}{ p value } \\
\hline & & & $<47.5$ & $\geqq 47.5$ & & $<4.25$ & $\geqq 4.25$ & \\
\hline \multirow[t]{2}{*}{ Age } & $<50$ & 51 & 14 & 37 & 0.774 & 34 & 17 & 0.635 \\
\hline & $\geqq 50$ & 51 & 15 & 36 & & 31 & 20 & \\
\hline \multicolumn{9}{|c|}{ FIGO stage } \\
\hline & I/II & 60 & 24 & 36 & $0.005^{*}$ & 33 & 27 & $0.025^{\star}$ \\
\hline & III/IV & 42 & 5 & 37 & & 32 & 10 & \\
\hline \multicolumn{9}{|l|}{ Grade } \\
\hline & G1/G2 & 58 & 25 & 33 & $0.003^{*}$ & 12 & 46 & 0.531 \\
\hline & G3 & 44 & 4 & 40 & & 14 & 30 & \\
\hline \multicolumn{9}{|c|}{ Histopathology } \\
\hline & Serous & 76 & 55 & 30 & 0.634 & 50 & 26 & 0.642 \\
\hline & Others & 28 & 14 & 8 & & 16 & 12 & \\
\hline \multicolumn{9}{|c|}{ LN metastasis } \\
\hline & No & 80 & 26 & 54 & 0.377 & 50 & 30 & $0.031^{\star}$ \\
\hline & Yes & 22 & 5 & 17 & & 18 & 4 & \\
\hline \multicolumn{9}{|c|}{ Malignant ascites } \\
\hline & No & 67 & 24 & 43 & $0.046^{*}$ & 35 & 32 & $0.011^{*}$ \\
\hline & Yes & 35 & 5 & 30 & & 30 & 5 & \\
\hline \multicolumn{9}{|c|}{ Residual mass } \\
\hline & $<1 \mathrm{~cm}$ & 81 & 26 & 55 & 0.255 & 51 & 30 & 0.147 \\
\hline & $\geqq 1 \mathrm{~cm}$ & 21 & 3 & 18 & & 15 & 6 & \\
\hline \multicolumn{9}{|c|}{ Platinum response } \\
\hline & Sensitive & 80 & 28 & 52 & $0.008^{*}$ & 48 & 32 & $0.033^{*}$ \\
\hline & Resistant & 22 & 3 & 19 & & 17 & 5 & \\
\hline
\end{tabular}

Note: FIGO, International Federation of Gynecology and Obstetrics; LN, lymph node. 
$\mathbf{A}$

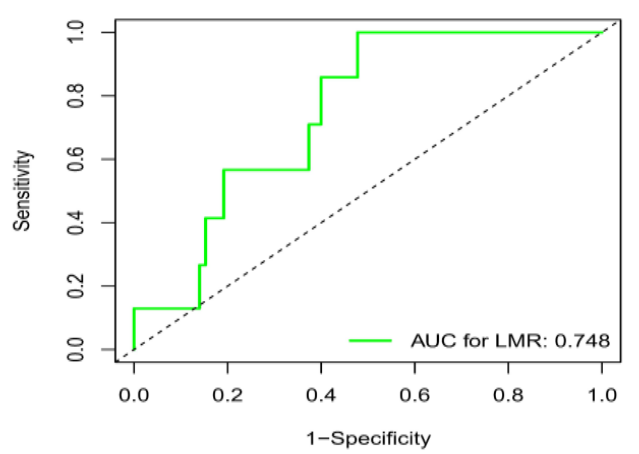

B

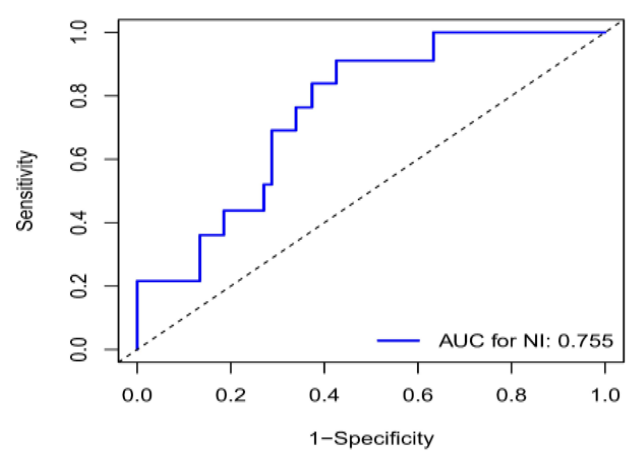

Figure 1: Receiver operating characteristic curves. Receiver operating characteristic curves for predicting the survival outcome. (A) Lymphocyte to Monocyte Ratio (LMR) (B) Nutritional Index (NI).

The histopathological type (76 patients) is mostly serous epithelial carcinoma. Eighty-one patients were optimally debulked with less than one $\mathrm{cm}$ of residual disease at primary surgery. Almost all patients are sensitive to platinum (Table 1).

\section{Relations between NI, LMR and Clinical Features}

The Chi-square test was used to evaluate the association between the levels of NI, LMR and clinical characteristics (Table 1), including Age, FIGO stage, Grade, Histopathology, LN metastasis, Malignant ascites, Residual mass, and Platinum response. NI was shown to be significantly correlated with FIGO stage $(\mathrm{P}=0.005)$, Grade $(\mathrm{P}=$ $0.003)$, malignant ascites $(P=0.046)$, and platinum response $(P=$ $0.008)$, and LMR with FIGO stage $(\mathrm{P}=0.025)$, lymph node metastasis $(\mathrm{P}=0.031)$, malignant ascites $(\mathrm{P}=0.011)$, and platinum response $(\mathrm{P}$ $=0.033)$ when the population was separated using optimized cut-off.

$\mathbf{A}$

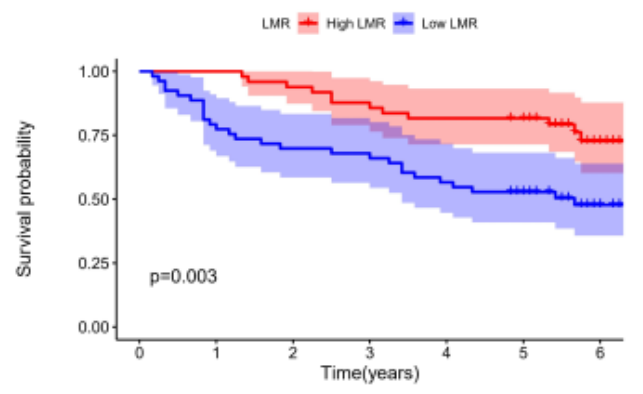

C

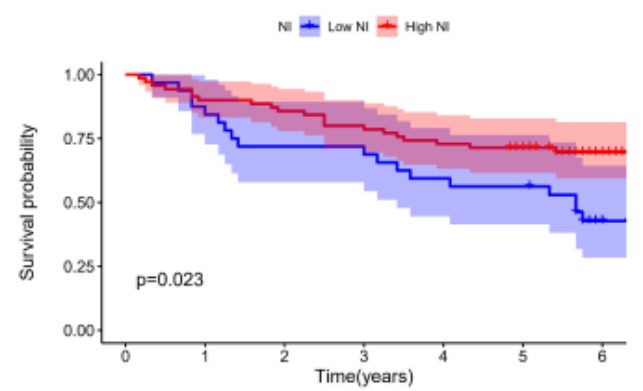

$\mathrm{KM}$ analysis found that patients in the low NI group had worse PFS $(P=0.023)$ and OS $(P=0.046)$ selected for this research than patients in the high NI group. In terms of PFS $(\mathrm{P}=0.003)$ and OS $(\mathrm{P}<0.016)$, patients in the high LMR group had greater treatment outcomes than those in the low LMR group, as shown in Figure 2.

\section{Prognostic Values of NI and MLR}

Univariate analyses showed that interactions with FIGO stage ( $\mathrm{P}$ $<0.001)$, Grade $(\mathrm{P}<0.001)$, LN metastasis $(\mathrm{P}=0.002)$, malignant ascites $(\mathrm{P}=0.003)$, histopathology $(\mathrm{P}=0.004)$, residual mass $(\mathrm{P}=$ $0.005)$, platinum reaction $(\mathrm{P}=0.009)$, NI $(\mathrm{P}=0.043)$ and LMR ( $\mathrm{P}$ $=0.004$ ) were identified in the findings obtained for PFS. Multiple cox regression analysis was used to analyze the association between survival outcomes and clinical features found in univariate analysis. A FIGO level (III-IV stage) (4.022 (1.754-9.322), P=0.001), grade III

\section{B}

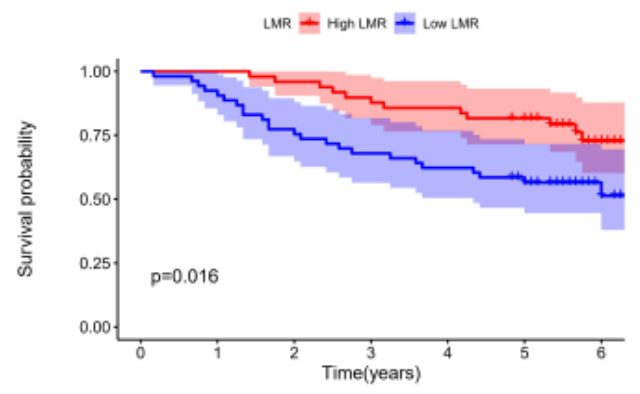

D

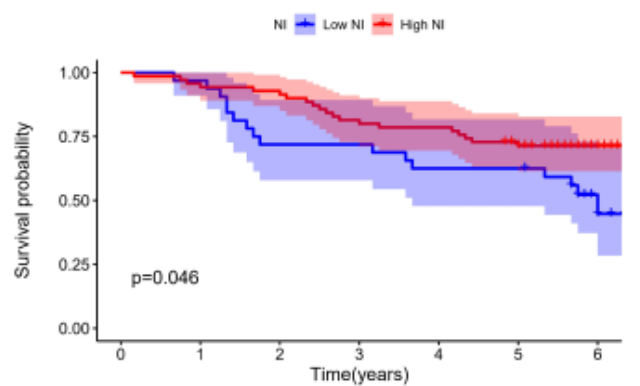

Figure 2: Kaplan-Meier survival curves. Kaplan-Meier survival curves by different level of LMR and NI. (A) LMR for Overall survival. (B) LMR for Progression-free survival. (C) NI for Overall survival. (D) NI for Progression-free survival. 
(2.640 (1.333-5.229), $\mathrm{P}=0.005)$, residual mass $1 \mathrm{~cm}$ (2.540 (1.0745.929), $\mathrm{P}=0.005)$ and immune platinum reaction (3.575 (2.672-6.752), $\mathrm{P}=0.009)$, a low $\operatorname{LMR}(2.640(2.364-5.731), \mathrm{P}=0.005)$ and a low $\mathrm{NI}$ (1.367 (0.243-2.254), $\mathrm{P}=0.019)$, as shown in Table 2 .

Likewise, univariate analysis showed important relationships between the following factors and OS: FIGO stage $(\mathrm{P}<0.001)$, Grade $(\mathrm{P}<0.001)$, LN metastasis $(\mathrm{P}=0.033)$, histopathology $(\mathrm{P}=0.014)$, residual mass $(\mathrm{P}=0.007)$, platinum reaction $(\mathrm{P}=0.001)$, NI $(\mathrm{P}=$ $0.034)$ and $\operatorname{LMR}(\mathrm{P}=0.001)$. However, COX multivariate analysis showed only the following were independent poor prognostic factor of OS, FIGO level (III-IV stage) (6.172 (2.315-10.112), P=0.003), grade III (3.640 (2.371-6.551), $\mathrm{P}=0.015)$, residual mass $1 \mathrm{~cm}(3.230$ (2.099-4.872), $\mathrm{P}=0.035)$ and immune platinum reaction (6.533 (3.2327.992), $\mathrm{P}=0.001)$, a low LMR (3.540 (2.724-6.133), $\mathrm{P}=0.002)$ and $\mathrm{a}$ low NI (1.667(0.349-3.692), $\mathrm{P}=0.014)$, as shown in Table 3.

\section{Discussion}

For women with OC, the high fatality risk is largely attributed to a lack of early diagnosis. There is no diagnosis for certain women until the late stage, so early diagnosis of OC is urgent. The main method used for ovarian cancer screening during physical examination is actually the concentration of serum CA125 monitoring. Furthermore, CA125 concentration can also be used to evaluate longevity following surgery in women who have been diagnosed with OC. Unfortunately, not all women with OC show high concentration of CA125. The low concentration of preoperative CA125 in OC patients was 20\% [13], according to a retrospective study. It indicated that certain patients were preoperative CA125-normal OC patients and that there was a lack of effective serum biomarkers to determine the prognosis.

LMR was elevated in epithelial ovarian cancer in pretreatment and showed prognostic importance after following treatment. Immune Complexes (ICs) are formed against the antigen by the antigen and antibody, and free ICs circulating are Circulating Immune Complexes (CICs) [14]. Any medium-sized CICs, however, cannot be washed and stay in the circulatory system. Inflammatory reaction, which is a central mechanism for immune-complex diseases, could be triggered by these CICs. Daniel demonstrated the presence of CICs affecting CA125 in 2010, and proposed that CA125 CICs offer a reason for ovarian cancer with normal CA125 level [15]. LMR should be a good predictor of ovarian cancer based on both of these results.

A low PNI demonstrated a decrease in serum albumin and/or a low lymphocyte absolute count. Serum albumin is an essential component

Table 2: Univariate and multivariate analyses of PFS of patients according to clinicopathological characteristics including LMR and NI.

\begin{tabular}{|c|c|c|c|c|}
\hline \multirow{2}{*}{ Variable } & \multicolumn{2}{|c|}{ Univariate } & \multicolumn{2}{|c|}{ Multivariate } \\
\hline & HR (95\% CI) & $\mathrm{p}$ value & HR $(95 \% \mathrm{CI})$ & p value \\
\hline Age ( $\geq 50$ vs. $<50)$ & $1.544(0.832-2.774)$ & 0.123 & & \\
\hline FIGO stage (III/IV vs. I/II) & $8.337(3.874-14.665)$ & $<0.001$ & $4.022(1.754-9.322)$ & $0.001^{*}$ \\
\hline Grade (III vs. I/II) & $6.367(2.575-10.254)$ & $<0.001$ & $2.640(1.333-5.229)$ & $0.005^{*}$ \\
\hline Histopathology (Others vs. Serous) & $4.723(3.687-7.263)$ & 0.004 & $1.224(0.875-3.354)$ & 0.479 \\
\hline LN metastasis (Yes vs. No) & $2.633(1.377-5.357)$ & 0.002 & $1.239(0.994-2.995)$ & 0.632 \\
\hline Malignant ascites (Yes vs. No) & $3.627(1.756-6.233)$ & 0.018 & $0.994(0.383-3.411)$ & 0.255 \\
\hline Residual mass ( $\geq 1 \mathrm{~cm}$ vs. $<1 \mathrm{~cm}$ ) & $4.540(1.383-5.929)$ & 0.015 & $2.540(1.074-5.929)$ & $0.005^{*}$ \\
\hline Platinum response (Resistant vs. Sensitive) & $10.367(7.575-12.254)$ & 0.009 & $3.575(2.672-6.752)$ & $0.009^{*}$ \\
\hline LMR (<4.25 vs. $\geq 4.25)$ & $3.066(1.432-6.229)$ & 0.004 & $2.640(2.364-5.731)$ & $0.005^{\star}$ \\
\hline NI $(\geq 47.5$ vs. $<47.5)$ & $1.795(1.575-4.254)$ & 0.043 & $1.367(0.243-2.254)$ & $0.019^{*}$ \\
\hline
\end{tabular}

Note: HRs was obtained from Cox s proportional hazard model. HR, hazard ratio; CI, confidence interval; NI, neutrophil lymphocyte ratio; LMR, lymphocyte monocyte ratio; FIGO, The International Federation of Gynecology and Obstetrics; LN, lymph node.

Table 3: Univariate and multivariate analyses of OS of patients according to clinicopathological characteristcs including LMR and NI.

\begin{tabular}{|c|c|c|c|c|}
\hline \multirow{2}{*}{ Variable } & \multicolumn{2}{|c|}{ Univariate } & \multicolumn{2}{|c|}{ Multivariate } \\
\hline & HR $(95 \%$ CI $)$ & p value & HR $(95 \%$ CI $)$ & p value \\
\hline Age ( $\geq 50$ vs. $<50)$ & $1.454(0.730-2.668)$ & 0.313 & & \\
\hline FIGO stage (III/IV vs. I/II) & $9.127(3.765-20.175)$ & $<0.001$ & $6.172(2.315-10.112)$ & $0.003^{*}$ \\
\hline Grade (III vs. I/II) & $7.367(3.723-12.557)$ & $<0.001$ & $3.640(2.371-6.551)$ & $0.015^{*}$ \\
\hline Histopathology (Others vs. Serous) & $3.436(2.227-8.923)$ & 0.014 & $2.367(1.445-3.674)$ & 0.331 \\
\hline LN metastasis (Yes vs. No) & $1.783(1.267-4.349)$ & 0.033 & $1.211(0.749-1.995)$ & 0.362 \\
\hline Malignant ascites (Yes vs. No) & $4.007(1.366-5.273)$ & 0.147 & & \\
\hline Residual mass $(\geq 1 \mathrm{~cm}$ vs. $<1 \mathrm{~cm})$ & $4.880(2.367-6.429)$ & 0.007 & $3.230(2.099-4.872)$ & $0.035^{*}$ \\
\hline Platinum response (Resistant vs. Sensitive) & $9.237(6.945-10.322)$ & 0.001 & $6.533(3.232-7.992)$ & $0.001^{*}$ \\
\hline LMR (<4.25 vs. $\geq 4.25)$ & $6.014(2.397-7.379)$ & 0.001 & $3.540(2.724-6.133)$ & $0.002^{*}$ \\
\hline NI ( $\geq 47.5$ vs. $<47.5)$ & $1.993(1.235-5.641)$ & 0.033 & $1.667(0.349-3.692)$ & $0.014^{*}$ \\
\hline
\end{tabular}

Note: HRs was obtained from Cox s proportional hazard model. HR, hazard ratio; CI, confidence interval; NI, neutrophil lymphocyte ratio; LMR, lymphocyte monocyte ratio; FIGO, The International Federation of Gynecology and Obstetrics; LN, lymph node. 
in the nutritional status and inflammatory response of the host [16]. It is often considered that the absolute lymphocyte count is a significant participant in inhibiting cancer growth by initiating a cytotoxic immune response [17]. It has been documented that low immune-nutritional status is associated with an immunosuppressed disorder that offers a favorable micro-environment for tumor relapse. That may be the reason why the bad results may be caused by this immunosuppressed syndrome in low-NI patients. Important advancement in research on immune control points in tumor immunity has made it possible to elucidate the molecular mechanism underlying the immunological resistance of tumor growth. The relation between peripheral inflammatory biomarkers and immunotherapy treatment effects appears to be uncertain. These biomarkers could serve in the future as a helpful indicator of immunotherapy in the treatment of OC.

Taken together, this current literature has demonstrated that a severely compromised immune system may be affected by starvation and lymphocytopenia. The NI cutoff value reported in previous studies was 40-60 for other cancer forms [18-20]. In our study, patients with NI $<47.5$ had dramatically decreased survival when multivariate regression was corrected for other prognostic factors. Furthermore, our findings have shown that low LMR is a predictor of poor prognosis in OC patients with average levels of CA125. According to the multivariate study, patients with LMR $<4.25$ had a substantial decrease in OS and PFS. Moreover, since the LMR and NI are very quickly collected, the cost-effectiveness is in line with the criteria of regular screening markers.

Any of the present study's limitations merit attention. First, we were unable to thoroughly validate the prognostic value of LMR and NI due to the retrospective aspect of the analysis. Second, LMR is a non-specific inflammation marker, and while we omitted patients with any inflammatory disorder, the existence of other unrecognized systemic inflammatory disorders could have impaired laboratory findings. The strength of our research is that it is the first attempt to evaluate the prognostic importance of LMR and NI in OC patients with normal CA125 level.

\section{Conclusion}

In summary, our current study showed that patients with higher pretreatment LMR ( $\geqq 4.25$ ) showed significantly better survival than those with lower LMR $(<4.25)$; Patients with higher NI $(\geqq 47.5)$ revealed pretreatment LMR and NI were also an independent prognostic factor that predicts OS and PFS.

\section{Declarations}

\section{Ethics Approval and Consent to Participate}

The authors are accountable for all aspects of the work in ensuring that questions related to the accuracy or integrity of any part of the work are appropriately investigated and resolved. The study protocol was approved by the Hospital Ethics Committee of the Second Affiliated Hospital of Soochow University.

\section{References}

1. Jelovac D, Armstrong DK (2011) Recent progress in the diagnosis and treatment of ovarian cancer. CA Cancer J Clin 61: 183-203. [crossref]

2. Pauler DK, Menon U, McIntosh M, Symecko HL, Skates SJ, et al. (2001) Factors influencing serum CA125II levels in healthy postmenopausal women. Cancer Epidemiol Biomarkers Prev 10: 489-493. [crossref]

3. Cramer DW, O'Rourke DJ, Vitonis AF, Matulonis UA, Dijohnson DA, et al (2010) CA125 immune complexes in ovarian cancer patients with low CA125 concentrations. Clin Chem 56: 1889-1892. [crossref]

4. Simmons AR, Fourkala EO, Gentry-Maharaj A, Ryan A, Sutton MN, et al. (2019) Complementary Longitudinal Serum Biomarkers to CA125 for Early Detection of Ovarian Cancer. Cancer Prev Res (Phila) 12: 391-400. [crossref]

5. Mason M, Maurice C, McNamara MG, Tieu MT, Lwin Z, et al. (2017) Neutrophillymphocyte ratio dynamics during concurrent chemo-radiotherapy for glioblastoma is an independent predictor for overall survival. J Neurooncol 132: 463-471. [crossref]

6. Wang W, Bian C, Xia D, et al. (2017) Combining Carcinoembryonic Antigen and Platelet to Lymphocyte Ratio to Predict Brain Metastasis of Resected Lung Adenocarcinoma Patients. Biomed Res Int 2017: 8076384. [crossref]

7. Suh KJ, Kim SH, Kim YJ, He JX, Hai P, et al. (2018) Post-treatment neutrophil-tolymphocyte ratio at week 6 is prognostic in patients with advanced non-small cell lung cancers treated with anti-PD-1 antibody. Cancer Immunol Immunother 67: 459470. [crossref]

8. Lu C, Zhou L, Ouyang J, Yang H (2019) Prognostic value of lymphocyte-to-monocyte ratio in ovarian cancer: A meta-analysis. Medicine (Baltimore) 98: e15876. [crossref]

9. Oba T, Maeno K, Ono M, Ito T, Kanai T, et al. (2021) Prognostic Nutritional Index Is Superior to Neutrophil-to-lymphocyte Ratio as a Prognostic Marker in Metastatic Breast Cancer Patients Treated with Eribulin. Anticancer Res 41: 445-452. [crossref]

10. Wang Z, Wang Y, Zhang X, Zhang T (2018) Pretreatment prognostic nutritional index as a prognostic factor in lung cancer: Review and meta-analysis. Clin Chim Acta 486: 303-310. [crossref]

11. Okadome K, Baba Y, Yagi T, Kiyozumi Y, Ishimoto T, et al. (2020) Prognostic Nutritional Index, Tumor-infiltrating Lymphocytes, and Prognosis in Patients with Esophageal Cancer. Ann Surg 271: 693-700. [crossref]

12. Jordan T, Mastnak DM, Palamar N, Kozjek NR (2018) Nutritional Therapy for Patients with Esophageal Cancer. Nutr Cancer 70: 23-29. [crossref]

13. Zhang H, Huo Q, Huang L, Cheng Y, Liu Y, Bao H (2019) Neutrophil-to-Lymphocyte Ratio in Ovarian Cancer Patients with Low CA125 Concentration. Biomed Res Int 2019: 8107906.

14. Yoshimoto T, Tanaka M, Homme M, Yamazaki Y, Takazawa Y, et al. (2017) CICDUX4 Induces Small Round Cell Sarcomas Distinct from Ewing Sarcoma. Cancer Res 77: 2927-2937. [crossref]

15. Cramer DW, O'Rourke DJ, Vitonis AF, Matulonis UA, Dijohnson DA, et al (2010) CA125 immune complexes in ovarian cancer patients with low CA125 concentrations. Clin Chem 56: 1889-1892. [crossref]

16. Mirili C, Yılmaz A, Demirkan S, Bilici M, Basol Tekin S (2019) Clinical significance of prognostic nutritional index (PNI) in malignant melanoma. Int J Clin Oncol 24: 1301-1310. [crossref]

17. Mantovani A, Allavena P, Sica A, Balkwill F (2008) Cancer-related inflammation. Nature 454: 436-444.

18. Salcedo MP, Sood AK, Dos Reis R, Ramalingam P, Chen C, et al. (2019) Perineural invasion (PNI) in vulvar carcinoma: A review of 421 cases. Gynecol Oncol 152: 101105. [crossref]

19. Shen Y, Li H, Yuan ZQ, et al. (2020) Low pretreatment PNI correlates with worse survival in patients with stage III/IV NSCLC who received chemotherapy. Neoplasma 67: 394-401. [crossref]

20. Lv X, Zhang Z, Yuan W (2020) Pretreatment Prognostic Nutritional Index (PNI) as a Prognostic Factor in Patients with Biliary Tract Cancer: A Meta-Analysis. Nutr Cancer 1-10. [crossref]

\section{Citation:}

Feng S, Luo S, Ji C, Li Y (2021) The Prognostic Value of Lymphocyte-to-Monocyte Ratio and Nutritional Index for Ovarian Cancer Patients with Normal CA125 Level. Cancer Stud Ther J Volume 6(1): 1-5. 